\title{
The Influence of a Restrictive Atrial Septal Defect on Pulmonary Vascular Morphology in Patients with Hypoplastic Left Heart Syndrome
}

\author{
J.N. Graziano, ${ }^{1}$ K.P. Heidelberger,${ }^{2}$ G. J. Ensing, ${ }^{1}$ C.A. Gomez, ${ }^{1}$ A. Ludomirsky ${ }^{1}$ \\ ${ }^{1}$ Department of Pediatrics, Division of Pediatric Cardiology, University of Michigan, Ann Arbor, MI 48109, USA \\ ${ }^{2}$ Department of Pathology, University of Michigan, Ann Arbor, MI 48109, USA
}

Abstract. Hypoplastic left heart syndrome (HLHS) with a restrictive atrial septal defect (ASD) is a form of congenital heart disease with considerable morbidity and mortality. This morphologic analysis assesses the pulmonary vasculature in this patient population. Pulmonary arteries, the persistence of high-resistance fetal arterioles, pulmonary veins, and lymphatics from multiple lung sections from each of five patients with HLHS and a restrictive ASD were compared to those of five patients with HLHS and nonrestrictive ASD. Lung sections from each patient were qualitatively graded in severity of pathology from 0 to 3 for each of the structures described previously, with the pathologist blinded to the status of the ASD. Patients with a restrictive ASD exhibited more significant pulmonary venous thickening and lymphatic dilatation $(p=0.02)$, with a tendency toward persistence of high-resistance fetal vessels ( $p=$ 0.2 ), compared to patients with a nonrestrictive ASD. These findings imply that patients with HLHS and a restrictive ASD possess pulmonary vascular abnormalities that place them at higher risk for the current surgical interventions available compared to patients with a nonrestrictive ASD.

Key words: Hypoplastic left heart syndrome Pulmonary vasculature - Atrial septal defect

Hypoplastic left heart syndrome (HLHS) is a form of congenital heart disease consisting of aortic and/or mitral atresia resulting in inadequate formation and development of a functional left ventricle. The con-

Correspondence to: A. Ludomirsky, University of Michigan Medical Center, Division of Pediatric Cardiology, F1310 MCHC, Box 0204, 1500 East Medical Center Drive, Ann Arbor, MI 48109-0204, USA dition in utero has little effect on the fetal circulation because the right ventricle is the predominant source of systemic blood flow via the ductus arteriosus. Furthermore, systemic oxygenation is independent of left ventricular output in utero because the supply of oxygenated blood is delivered to the right heart via the umbilical vein. Therefore, the absence of a left ventricle has essentially no detrimental effect on fetal circulation and hemodynamics.

Although blood flow via the pulmonary circulation has been described to be minimal, accounting for $3 \%$ to $7 \%$ of the total right ventricular output [9], it represents flow that returns to the left atrium. In patients with mitral atresia or severe mitral stenosis, decompression of this return of flow to the left atrium must occur via the foramen ovale. The absence of an adequate-sized foramen ovale likely results in left atrial hypertension in utero, which is transmitted to the pulmonary venous structures with resultant pulmonary venous hypertension. It therefore seem plausible that restriction of obligatory left-to-right blood flow at the level of the atrial septum in patients with HLHS would have deleterious consequences on pulmonary vascular development.

Pulmonary vascular maldevelopment has significant implications in HLHS because the current palliative interventions necessitate a low pulmonary resistance to ensure adequate pulmonary blood flow via aortopulmonary shunts (i.e., Norwood procedure) $[11,17]$ and, ultimately, cavopulmonary (i.e., hemi-Fontan and Fontan) connections [2, 16]. Furthermore, cardiac transplantation remains a potential treatment modality [4], the success of which is optimized by normal pulmonary vasculature to prevent post transplantation right ventricular failure. The purposes of this study were to evaluate the pulmonary vascular morphology in neonates with HLHS and to determine if a restrictive atrial septal defect (ASD) is associated with more severe pulmonary vascular changes. We hypothesize that patients with 
Table 1. Patient characteristics

\begin{tabular}{|c|c|c|}
\hline Characteristic & Nonrestrictive ASD $(n=5)$ & Restrictive ASD $(n=5)$ \\
\hline Sex (Male:Female) & $2: 3$ & $1: 4$ \\
\hline Cardiac pathology (MA/AA:MS/AA) & $4: 1$ & $3: 2$ \\
\hline Birth weight $(\mathrm{kg})$ & $2.73 \pm 0.48$ & $2.67 \pm 0.75$ \\
\hline Gestational age (weeks) & $39.2 \pm 1.5$ & $38.8 \pm 1.9$ \\
\hline Preoperative deaths & 1 (3 days of age) & 2 (2 and 4 days of age) \\
\hline Age at Norwood (days) & $8.6 \pm 2.5$ & $7.0 \pm 4.2$ \\
\hline Age of death (days) & $9.4 \pm 3.1$ & $11.2 \pm 5.0$ \\
\hline Postoperative deaths & 4 ( $0-3$ days postop) & 3 (0-16 days postop) \\
\hline
\end{tabular}

$p>0.05$ for each of the clinical parameters, indicating no significant difference between the two groups. Data are presented as mean \pm SEM or as number of patients. AA, aortic atresia; MA, mitral atresia; MS, mitral stenosis.

restriction of left-to-right blood flow at the level of the atrial septum demonstrate more severe pulmonary vascular changes.

\section{Methods}

We retrospectively reviewed the echocardiography database from January 1990 through September 1998 at the University of Michigan Congenital Heart Center for patients with HLHS and normal pulmonary venous return to the left atrium. HLHS was defined as the presence of aortic and/or mitral atresia with a hypoplastic left ventricle. Patients were then classified into two groups according to the status of the atrial septum: First, a restrictive ASD was defined as a mean spectral Doppler gradient of $8 \mathrm{mmHg}$ or more from left atrium to right atrium, across the atrial septum. The mean gradient was measured during the entire cardiac cycle, over three to five consecutive beats, without the use of angle correction, generally from the subcostal views to best align the jet of left-to-right flow. A gradient of $8 \mathrm{mmHg}$ was arbitrarily chosen and agreed upon by all the authors because it was believed to be a level of restriction that was likely to be clinically significant and greater than that solely attributable to excessive pulmonary blood flow. Second, a nonrestrictive ASD was defined as a mean spectral Doppler gradient of 3 $\mathrm{mmHg}$ or less across the atrial septum. Excluded were patients with a mean gradient between 3 and $8 \mathrm{mmHg}$ because this is a range of restriction that was believed to be influenced by the degree of pulmonary blood flow. Also excluded were patients with an intact atrial septum because this population of patients can often have alternative decompression pathways from the left atrium, thereby making determination of the left-to-right atrial gradient difficult secondary to this decompression [1].

The pathology database of postmortem examination specimens was also reviewed and the five most recent specimens of patients with HLHS and a restrictive ASD, as defined previously from the echocardiography database, were chosen for pathologic review of their pulmonary vasculature. The five most recent postmortem examinations of patients with HLHS and a nonrestrictive ASD were also selected for pathologic review and comparison of their pulmonary vasculature.

Slides of pathologic specimens were qualitatively examined with the pathologist blinded to the status of the atrial septum. Three to six lung sections were reviewed from each lung for each patient. At least one section from each lung was taken through the hilum to ensure adequate assessment of the pulmonary venous morphology. Sections were examined with H\&E and elastic von Gieson stains and were assessed for the following characteristics:

1. Pulmonary arterioles were assessed for the degree of medial hypertrophy and were graded from 0 (normal) to 3 (marked hypertrophy with muscular extension to the smaller arterioles).

2. Arteriolar morphology was also examined for the presence and frequency of persistent fetal vessels. These are small, hypertrophied, high-resistance vessels that often appear as a "knot-like" configuration on microscopic examination. They are usually present at birth and generally resolve over the course of a few days. Their persistence and abundance are often indicative of elevated pulmonary artery pressures and are abnormal [6]. The frequency of these vessels was graded from 0 (no vessels noted) to 3 (vessels frequently seen in all the sections examined).

3. Pulmonary veins were assessed for the degree of intimal fibrosis and proliferation and were graded from 0 (normal) to 3 (markedly thickened intima with near obstruction of the vessel lumen).

4. The lymphatic system was assessed and graded for the severity of dilatation. The grade of severity between the two groups was compared using Wilcoxon rank-sum tests to determine statistical significance for the differences.

\section{Results}

Demographic data for the patients in nonrestrictive and restrictive ASD groups are shown in Table 1. No significant difference between them was noted with regard to male-to-female ratio, mean birth weight, mean gestational age, number of preoperative deaths, age at the Norwood procedure, age at death, number of postoperative deaths, and postoperative time until death. No patient in either group underwent a preoperative atrial septostomy/septectomy, nor did any of the patients have a decompression pathway for left atrial egress.

The causes of death in the three preoperative patients were an abrupt hypotensive episode with an unsuccessful resuscitation in one patient (nonrestrictive ASD) and the withdrawal of support at request 


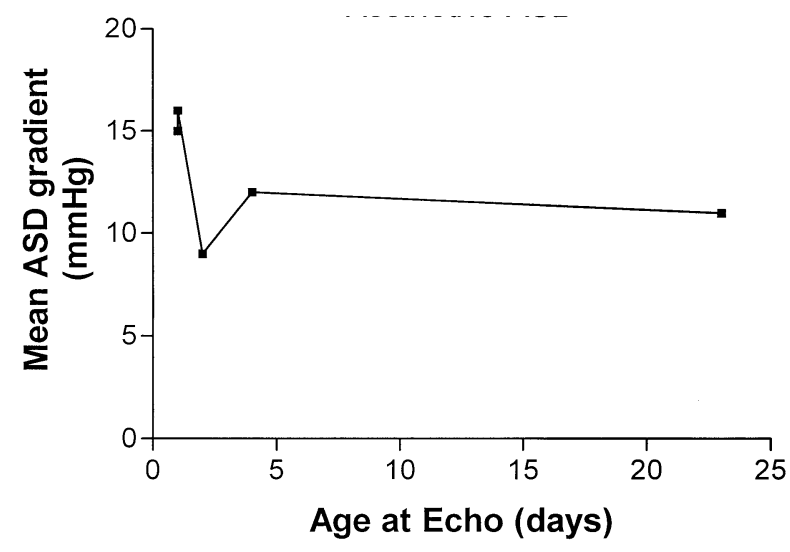

Fig. 1. The age of each patient with HLHS and a restrictive ASD at the time of the echocardiogram is plotted with the corresponding mean gradient across the atrial septal defect.

of the families due to severe hypoxemia and hemodynamic instability in two patients (restrictive ASD). Of the seven postoperative deaths, six were due to sudden episodes of hypotension and bradycardia with unsuccessful resuscitative efforts. One patient experienced an episode of unstable ventricular tachycardia with an unsuccessful resuscitation.

Because the natural decrease in pulmonary vascular resistance during the first few days of life results in increased pulmonary blood flow, the gradient across the ASD can potentially be influenced by the age of the patient when the measurement was obtained. Figure 1 demonstrates that despite the range in age at which the echocardiograms were obtained in the patients with restrictive ASDs (range $=1-23$ days), the mean gradients across the atrial septa were relatively constant and significantly elevated to cause at least moderate left atrial hypertension in all five patients. Furthermore, the two highest gradients (15 and $16 \mathrm{mmHg}$ ) were obtained at 1 day of age and four of the five patients were studied at less than 5 days of age, suggesting that older age and increased pulmonary blood flow were not solely responsible for the substantially elevated gradients in these patients. There was no correlation between ASD size as measured by echocardiogram (range $2.0-2.9 \mathrm{~mm}$ ) and mean gradient across the defect in patients with restrictive ASDs, and all five patients had continuous left-to-right flow throughout the cardiac cycle.

Histologic assessment with the pathologist blinded to the status of the atrial septum demonstrated abnormalities of the pulmonary arterioles in 7 of the 10 patients. Moderate pulmonary arteriole thickening was seen in 3 patients in each of the two groups with no significant difference between the groups (Fig. 2A; $p=0.40$ ). The frequency of persistent fetal vessels was abnormal in 9 of the 10 pa- tients, with a slightly higher frequency of persistence in the restrictive ASD group (Fig. 2B; $p=0.12$ ). Although 3 patients in the restrictive ASD group were 4 days old or younger, 2 of these patients in fact had a lower frequency of fetal vessels than the 2 older patients who died at ages 23 and 24 days (Fig. 2B).

Pulmonary venous abnormalities were observed in 7 of the 10 patients. All patients in the restrictive ASD group demonstrated abnormal venous thickening and fibrosis, whereas only 2 patients in the nonrestrictive group demonstrated abnormal pulmonary veins (Fig. 2C; $p=0.02$ ). Furthermore, 4 of the 5 patients in the restrictive ASD group had at least grade 2 venous thickening and 1 patient had severe (grade 3 ) venous thickening and fibrosis with near obstruction of the majority of veins. The severity of lymphatic abnormalities mimicked those of the pulmonary veins. Four of the 5 patients with a restrictive ASD demonstrated moderate lymphatic dilatation and 1 patient had severe dilatation. In contrast, 4 of the 5 patients with nonrestrictive defects had normally lymphatics and 1 patient had mildly dilated lymphatics $(p=0.02)$.

\section{Discussion}

Previous morphologic analyses of the pulmonary vasculature have repeatedly demonstrated abnormalities of the pulmonary vascular bed in patients with hypoplastic left heart syndrome [7, 8, 14]. These abnormalities have generally been described as affecting the pulmonary arteries and consist of increased arterial medial thickness with muscular extension to the smaller respiratory bronchioles. Early observations suggested more severe abnormalities in patients with closure of the foramen ovale consisting of "intralobular pulmonary artery tortuosity" and "pulmonary vein elastic hyperplasia" [7]. Decompression pathways from the left atrium to the superior vena cava or right atrium have been observed in this population as well, making determination of the degree of obstruction difficult solely on the basis of the anatomic appearance of the atrial septum [1]. A nearly intact atrial septum with an adequate decompression vessel may in fact not result in significant left atrial hypertension. Rychik et al. [18] classified patients with HLHS and intact atrial septum according to atrial septal morphology, with many patients demonstrating decompression pathways. Of those patients with obstructed decompression pathways, pulmonary arterial histopathology was described as being no different from that of patients with HLHS and a widely patent ASD, whereas pulmonary venous histopathology was described as 
A

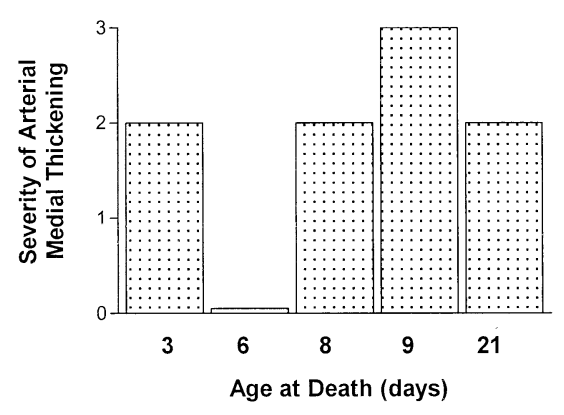

Restrictive ASD

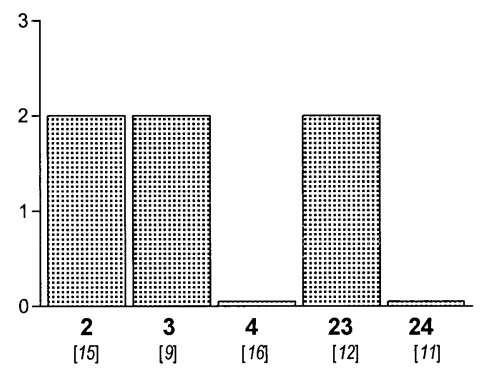

Age at Death (days)

[Mean ASD Gradient ( $\mathrm{mmHg}$ )]
Non-restrictive ASD

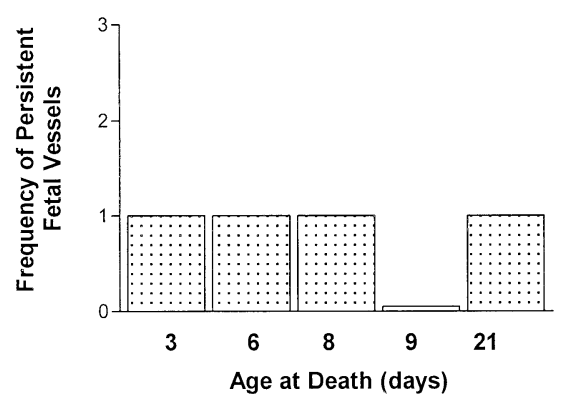

Restrictive ASD

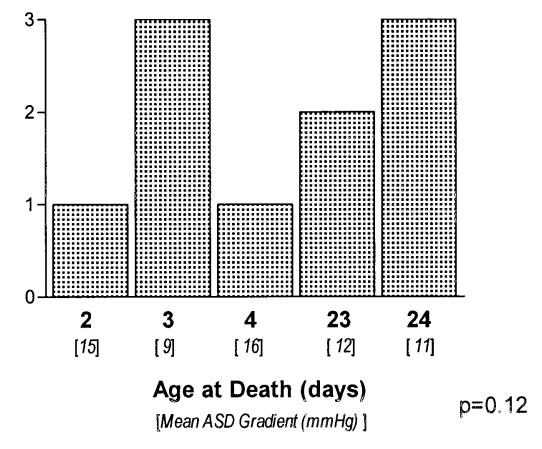

C Non-restrictive ASD

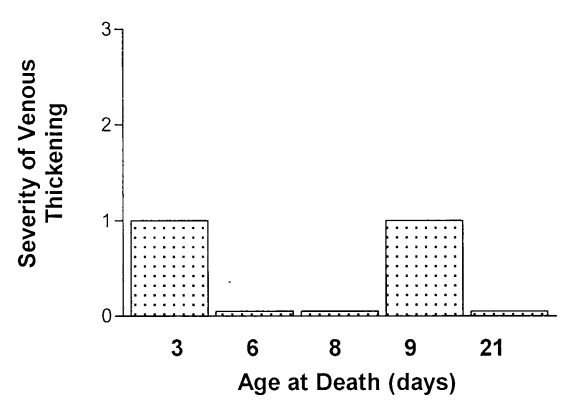

Restrictive ASD

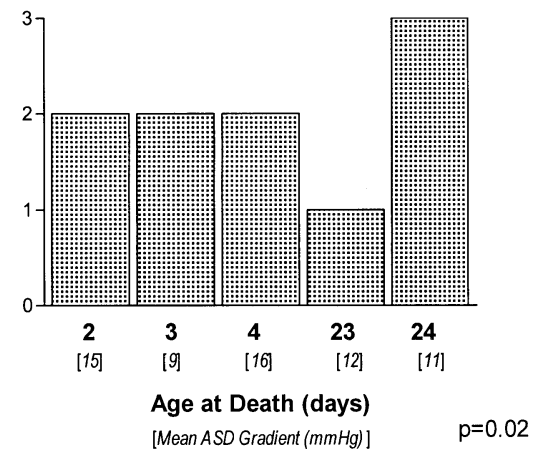

Fig. 2. (A) Severity of pulmonary arteriolar medial thickening in the nonrestrictive ASD and restrictive ASD groups $(p=0.40)$. (B) Frequency of persistent fetal vessels in the nonrestrictive ASD and restrictive ASD groups $(p=0.12)$. (C) Severity of pulmonary venous thickening in the nonrestrictive ASD and the restrictive ASD groups $(p=0.02)$.

thick and dilated with markedly dilated lymphatics, more severe than in the control group.

Echocardiographic measurement of a mean gradient from left atrium to right atrium across a restrictive ASD provides quantitative evidence for left atrial hypertension without the confounding factor of a decompression pathway and therefore serves as a model for determining the effects of increased left atrial pressure on pulmonary vascular development in this group of patients. Our findings indicate that inadequate left atrial decompression has mild effects on pulmonary arteriole development 
with a dramatic consequence on pulmonary venous and lymphatic development. Luciani et al. [13], in a report of a single patient with congenital pulmonary lymphagiectasis and hypoplastic left heart syndrome with a restrictive ASD, likely described the most severe spectrum of the lymphatic abnormalities described in this study.

However, the long-term effects that these venous and lymphatic changes have on long-term outcome and whether the histopathologic abnormalities resolve are unknown. Long-term pulmonary pathologic data for patients with HLHS and restrictive ASDs are lacking. However, an analogous physiologic condition in utero is that of the patient with obstructed total anomalous pulmonary venous connection (TAPVC) with resultant pulmonary venous hypertension. Congenital pulmonary lymphangiectasis has been described in patients with obstructed TAPVC [5], similar to the case report by Luciani et al. [13]. Furthermore, histopathologic analysis of lung specimens from older infants following neonatal repair of obstructed TAPVC has demonstrated "involvement of the pulmonary veins with medial hypertrophy, intimal hypertrophy, and fibrosis," even in patients as old as 6 month of age [15]. These data suggest that the pulmonary venous abnormalities may persist at least until the typical time of the second palliation with the hemi-Fontan procedure.

Rychik et al. [18] reported a poor outcome for patients with HLHS and a restrictive ASD following the Norwood procedure. Although based on a small group of patients, their data suggest that patients surviving the Norwood procedure have a relatively higher morbidity and mortality following cavopulmonary connections, consistent with the fact that these pulmonary vascular abnormalities may indeed persist. Furthermore, animal models have shown hyperreactivity of the pulmonary vasculature with episodes of vasospasm after a period of in utero pulmonary venous hypertension, thereby potentiating any lability in the pulmonary vascular resistance and predisposing to episodic pulmonary hypertensive crises [19]. The abnormalities of the pulmonary vasculature demonstrated in this study and the probable vasospastic component of the vasculature certainly pose a theoretical risk for patients ultimately requiring a passive pulmonary circulation.

Severe cyanosis in the immediate neonatal period in these patients obviously necessitates immediate intervention with a surgical atrial septectomy or balloon septostomy to ensure survival. Interestingly, a recent survival analysis found no significant increase in mortality in patients who underwent atrial septostomy prior to the Norwood procedure [10], which is contradictory to anecdotal experience and data from earlier analyses [12, 18]. However, Canter et al. [3] have shown patients with HLHS and a restrictive ASD to be at significantly higher risk for death following transplantation compared to infants with HLHS without a restrictive ASD. Regardless of survival beyond the Norwood procedure, our morphologic analysis implies that pulmonary vascular abnormalities posing a significant physiologic risk for later cavopulmonary connections or transplantation are present in essentially all patients with HLHS and a restrictive ASD. Therefore, it is plausible to assume that mortality for this condition in the near future, despite survival beyond the Norwood procedure, will remain high without a good option for transplantation or further cavopulmonary palliation.

\section{References}

1. Bernstein HS, Moore P, Stanger P, Silverman NH (1995) The levoatriocardinal vein: morphology and echocardiographic identification of the pulmonary-systemic connection. $J \mathrm{Am}$ Coll Cardiol 26:995-1001

2. Bove E (1998) Current status of staged reconstruction for hypoplastic left heart syndrome. Pediatr Cardiol 19:308315

3. Canter CE, Moorehead S, Huddleston CB, Spray TL (1993) Restrictive atrial septal communication as a determinant of outcome of cardiac transplantation for hypoplastic left heart syndrome. Circulation 88:II456-II460

4. Chiavarelli M, Gundry SR, Razzouk AJ, Bailey LL (1993) Cardiac transplantation for infants with hypoplastic left heart syndrome. J Am Med Assoc 270:2944-2947

5. France NE, Brown RJK (1971) Congenital pulmonary lymphangiectasis: report of 11 examples with special reference to cardiovascular findings. Arch Dis Child 46:528-532

6. Geggel RL, Reid LM (1984) The structural basis of PPHN. Clin Perinatol 2:525-549

7. Grant CA, Robertson B (1972) Microangiography of the pulmonary arterial system in hypoplastic left heart syndrome. Circulation 45:382-388

8. Haworth SG, Reid L (1977) Quantitative structural study of pulmonary circulation in the newborn with aortic atresia, stenosis, or coarctation. Thorax 32:121-128

9. Heyman MA (1995) Fetal and postnatal circulations: pulmonary circulation. In: Emmanouilides GC, Riemenschneider TA, Allen HD, Gutgesell HP (eds) Moss and Adams' Heart Disease in Infants, Children, and Adolescents, 5th edn. Williams \& Wilkins, Baltimore, pp 41-47

10. Jenkins PC, Flanagan MF, Jenkins KJ, et al (2000) Survival analysis and risk factors for mortality in transplantation and staged surgery for hypoplastic left heart syndrome. $J$ Am Coll Cardiol 36:1178-1185

11. Jonas RA, Lang P, Hansen D, Hickey P, Castaneda AR (1986) First stage palliation for hypoplastic left heart syndrome. $J$ Thorac Cardiovasc Surg 92:6-13

12. Lloyd TR (1996) Prognosis of the hypoplastic left heart syndrome. Progr Pediatr Cardiol 5:57-64

13. Luciani GB, Pessotto R, Mombello A, Mazzucco A (1999) Hypoplastic left heart syndrome with restrictive atrial septal 
defect and congenital pulmonary lymphangiectasis. Cardiovasc Pathol 8:49-51

14. Neumann MP, Heidelberger KP, Dick M, Rosenthal A (1980) Pulmonary vascular changes associated with hypoplastic left heart syndrome. Pediatr Cardiol 1:301-306

15. Newfeld EA, Wilson A, Paul MH, Reisch JS (1980) Pulmonary vascular disease in total anomalous pulmonary venous drainage. Circulation 61:103-109

16. Norwood WI, Jacobs ML (1993) Fontan's procedure in two stages. Am J Surg 166:548-551
17. Norwood WI, Lang P, Hansen DD (1983) Physiologic repair of aortic atresia-hypoplastic left heart syndrome. $N$ Engl $J$ Med 308:23-26

18. Rychik J, Rome JJ, Collins MH, DeCampli WM, Spray TL (1999) The hypoplastic left heart syndrome with intact atrial septum: atrial morphology, pulmonary vascular histopathology and outcome. J Am Coll Cardiol 34:554-560

19. Silove ED, Tavenor WD, Berry CL (1972) Reactive pulmonary arterial hypertension after pulmonary venous constriction in the calf. Cardiovasc Res 6:36-44

DOI: $10.1007 / \mathrm{s} 00246-001-0063-6$

\section{Around PediHeart: Plastic Bronchitis}

Over the past year or two there have been a number of interesting cases submitted to the PediHeart bulletin board regarding a phenomena referred to in the literature and by PediHeart members as "plastic bronchitis". I felt that this would be a good time for Around PediHeart to summarize those postings. When associated with congenital heart disease "plastic bronchitis" is characterized by acellular fibromucinous bronchial casts that cause symptomatic airway obstruction. Similar casts are also seen conditions that cause inflammation of the bronchopulmonary tree but these casts tend to be made up of densely packed inflammatory (eosinophils) cells [1].

There were 8 cases presented to the group. No cardiologist had more than one case. All instances of plastic bronchitis occurred after a lateral tunnel type Fontan procedure with fenestration. For most patients the onset of symptoms was roughly 2--3 years after the Fontan. For one patient it began 6 months post Fontan. Three members included hemodynamic data. In one, the mean Fontan pressure was $13 \mathrm{mmHg}$ with a wedge pressure of $10 \mathrm{mmHg}$ and a left ventricular end diastolic pressure (LVEDP) of $5 \mathrm{mmHg}$. This patient was 6 months post Fontan and no therapy for the plastic bronchitis was successful including mucolytics, and Coumadin. The second case with available data was 18 months post-op and had a Fontan pressure of $13 \mathrm{mmHg}$ and an LVEDP of $8 \mathrm{mmHg}$. Again no treatment was successful. The last case with data was several years post-op from his Fontan. He had a mean Fontan pressure of 16--18 $\mathrm{mmHg}$ and an LVEDP of $8 \mathrm{mmHg}$. In this instance resolution of plastic bronchitis occurred with aggressive medical treatment using continuous outpatient Milrinone infusion to improve ventricular function and lower the Fontan mean pressure. While hemodynamic data was not available for the other cases one had hypoalbuminemia and another had heart failure and protein losing enteropathy a year after presenting with plastic bronchitis. In another case the plastic bronchitis occurred twice after two subsequent non-cardiac surgeries years apart. Treatments temporally associated with improvement included Milrinone infusion, thoracic duct ligation, steroid administration, cardiac transplantation, heparinization, and doing nothing. Treatment failures included anticoagulation therapy with Coumadin, coil embolization of pulmonary collateral vessels, inhaled mucolytics and bronchodilators, and doing nothing.

Plastic bronchitis is a real entity and, given that eight cardiologists from different centers posted a case on PediHeart, it may not be so very rare. The mechanism for plastic bronchitis may involve an elevation in systemic venous or pulmonary venous pressure [2]. This was the presumed cause behind the patient who improved with Milrinone therapy and perhaps the patient who later developed protein losing enteropathy and heart failure. It may also involve a primary abnormality in lymphatic drainage as suggested in the literature [1] and by the patient who improved after thoracic duct ligation. Finally, given that in some patients plastic bronchitis occurred and spontaneously resolved without reason or intervention, it would be safe to say that, while this condition is interesting, the jury is still out.

Francis McCarey, M.D. PediHeart Editor

\section{References}

1. Languepin J, Scheinmann P, Mahut B, et al. (1999) Bronchial casts in children with cardiopathies: the role of pulmonary lymphatic abnormalities. Pediatr Pulmonol 28:329-336

2. Quasney MW, Orman K, Thompson J, et al. (2000) Plastic bronchitis occurring late after the Fontan procedure: treatment with aerosolized urokinase. Crit Care Med 28:2107-2111 